This is a postprint version of the following published document:

De la Fuente, A. ; Lentisco,C. M. ; Bellido, L. ; Pérez Leal, R. ; Pastor E. and García Armada, A. (2015). "Analysis of the impact of FEC techniques on a multicast video streaming service over LTE", in IEEE. Networks and Communications (EuCNC), 2015 European Conference on pp.224-228.

DOI:10.1109/EuCNC.2015.7194072

(C) 2015. IEEE. Personal use of this material is permitted. Permission from IEEE must be obtained for all other uses, in any current or future media, including reprinting/republishing this material for advertising or promotional purposes, creating new collective works, for resale or redistribution to servers or lists, or reuse of any copyrighted component of this work in other works. 


\title{
Analysis of the Impact of FEC Techniques on a Multicast Video Streaming Service over LTE
}

\author{
Alejandro de la Fuente*, Carlos M. Lentisco ${ }^{\dagger}$, Luis Bellido ${ }^{\dagger}$, \\ Raquel Perez Leal ${ }^{*}$, Encarna Pastor $^{\dagger}$, Ana Garcia Armada* \\ *Department of Signal Theory and Communications (Universidad Carlos III de Madrid) \\ ${ }^{\dagger}$ Department of Telematics Engineering (Universidad Politécnica de Madrid) \\ Email: afuente@ tsc.uc3m.es*, clentisco@dit.upm.es ${ }^{\dagger}$
}

\begin{abstract}
In a multicast video streaming service the same multimedia content is sent to a mass audience using only one multicast stream. In multicast video streaming over a cellular network, due to the nature of the multicast communication, from a source to multiple recipients, and due to the characteristics of the radio channel, different for each receiver, transmission errors are addressed at the application level by using Forward Error Correction (FEC) techniques. However, in order to protect the communication over the radio channel, FEC techniques are also applied at the physical layer. Another important technique to improve the communication of the radio channel is the use of a single-frequency network. This paper analyzes the performance of a video streaming service over a cellular network taking into account the combined impact of different factors that affect the transmission, both the physical deployment of the service and the two levels of FEC.
\end{abstract}

\section{INTRODUCTION}

The growing demand for video services in mobile networks poses new challenges in the design of techniques to improve the throughput and the delays to provide those services. These techniques must guarantee the scalability for large amount of users and reliable transmission to everyone, every time and everywhere.

In the context of cellular networks, Evolved Multimedia Broadcast and Multicast Service (eMBMS) [1], defined by 3rd Generation Partnership Project (3GPP) for Long Term Evolution (LTE), provides a point-to-multipoint service that allows data transmissions from a single source to multiple recipients. This technique improves the scalability of broadcast and multicast transmissions in mobile networks. Furthermore, Multicast/Broadcast over Single Frequency Network (MBSFN) was proposed to improve the performance of Multimedia Broadcast and Multicast Service (MBMS) [1]. MBSFN outperforms the quality of the signal in the areas where there is overlapping coverage from different base stations, thus improving the performance when the User Equipment (UE) is placed in the cell edge.

However, while using multicast transmissions improves the efficient utilization of network resources, it is not possible to adjust transmission parameters such as the Modulation and Coding Scheme (MCS) according to the channel conditions for each receiver, as it is done for unicast transmissions. In multicast transmissions, the MCS is set by upper layers [2].

The choice of MCS implies a specific level of protection against errors, since the MCS defines a specific modulation scheme and also the Forward Error Correction (FEC) overhead that is applied at the physical layer. However, the radio channel conditions vary among all the users receiving a multicast service. Therefore, the Block Error Rate (BLER) of the users that receive the video service sent with a unique MCS can have a great variance. In order to increase the robustness and the reliability of the multicast transmissions, eMBMS proposes an additional level of FEC redundancy at the application layer [3]. If the Application Layer - Forward Error Correction (ALFEC) is not enough to recover a piece of information due to a high error rate, the application layer at a receiver can choose to request a unicast retransmission.

The solution proposed by 3 GPP to deliver video streaming over MBMS uses the File Delivery over Unidirectional Transport (FLUTE) protocol (over UDP) to send video segments with the corresponding AL-FEC over multicast. If the ALFEC decoder is not able to recover the video segment, a unicast retransmission is requested using Hypertext Transfer Protocol (HTTP). In order to facilitate this combination of multicast transmission/unicast recovery, video segments are generated following the Dynamic Adaptive Streaming over HTTP (DASH) recommendation.

There are several works that analyze the use of AL-FEC for eMBMS . In [4] a single-cell scenario is simulated to research how the FEC overhead can vary according to the cell range and the number of satisfied users. Other works analyze the system trade-off between the AL-FEC and Physical Layer - Forward Error Correction (PHY-FEC). In [5] a 19-cell MBSFN area is used to evaluate the trade-off between AL-FEC overhead and protection period and the MCS used at physical layer. It presents different combinations of these parameters to achieve the maximum service data rate both for pedestrian and vehicular users.

This paper investigates a new perspective analyzing the trade-off between AL-FEC and PHY-FEC for achieving the maximum service data rate while limiting the percentage of unicast retransmissions. The analysis of several scenarios where the MBSFN area is composed of different number of Evolved Node B (eNodeB)s, presents the advantages of the 7-cell and 19-cell MBSFN area deployments over a singlecell MBSFN area. In addition, the performance for different Intersite Distance (ISD) deployments are presented.

The rest of the paper is organized as follows. In Section 
II, the FEC schemes specified for LTE eMBMS streaming are described. The system model used in the simulations is detailed in Section III. The performance evaluation results are presented in Section IV. Finally, in Section V, the conclusions are explained.

\section{FEC SCHEMES FOR EMBMS STREAMING}

\section{A. Physical layer}

3GPP standard [2] defines Turbo codes as the PHY-FEC used to protect data against errors in the transmission over unreliable or noisy communication channels, such as the wireless channel. The eNodeB uses an adaptive modulation and coding scheme according to the radio channel conditions to optimize unicast transmissions, whereas, in multicast transmissions the MCS is fixed to all the users in the MBSFN area.

In order to prevent channel errors, a 24-bit Cyclic Redundancy Check (CRC) is calculated for and appended to each transport block. Thus, the CRC allows the receiver to detect the errors in the decoded transport block.

The downlink data modulation maps the block of bits to the associated set of modulation symbols. The modulation schemes supported for the LTE downlink include Quadrature Phase Shift Keying (QPSK), 16-Quadrature Amplitude Modulation (QAM), and 64-QAM, corresponding to two, four, and six bits per modulation symbol respectively.

\section{B. Application layer}

AL-FEC is an error correction technique that sends redundant data to facilitate the recovery of the lost packets. Currently, there is a wide range of proposed FEC techniques. In this paper, the analysis is limited to the use of Raptor codes, which is the solution standardized by 3GPP for transmissions over MBMS [3] to ensure reliable transmission over unreliable channels.

Raptor codes are fountain codes, coding on-the-fly as many symbols as necessary from the $k$ symbols of the source block. The decoder is able to recover the block if it receives a number of symbols slightly greater than $k$. Furthermore, 3GPP defines the use of systematic Raptor codes, which means that in the transmission of each video segment, the original source symbols are sent together with the generated repair symbols. Each block, independently encoded, is composed of $k$ symbols of payload, where the symbol size is constant inside the block. Following, $r$ repair symbols are generated and added to the $k$ source symbols. Thus, each user decodes the video according to the amount of redundancy introduced at the transmitter, and the symbols received. The code rate, defined as the ratio between the $k$ original symbols and the $k+r$ symbols resulting of the encoding process is normally used to represent the amount of redundancy introduced at the transmitter. In wireless environments, with limited resources and high packet loss, it is necessary to find the value of the code rate that maximizes the useful data rate while guaranteeing a target coverage [6].

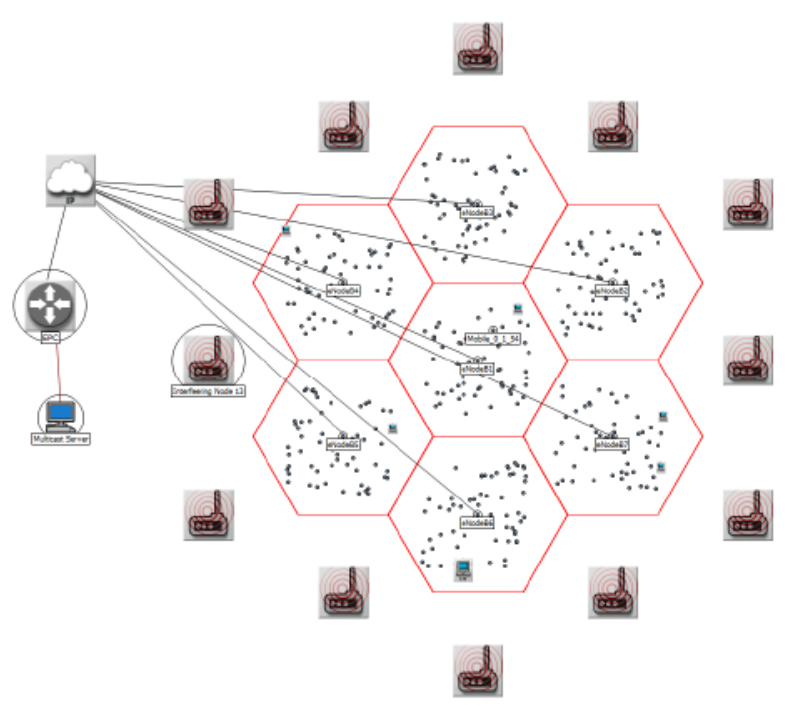

Fig. 1. System model scenario for 7-cell MBSFN area

\section{SYSTEM MODEL}

For this analysis, an LTE system is configured, using different MBSFN area sizes for multicast transmissions. A multicast service is delivered using one reserved subframe for MBMS to all the UEs placed in the region. Around the MBSFN area, we consider one tier of eNodeBs operating with the same frequency and transmission power as the eNodeBs in the MBSFN area. The system model scenario for the 7-cell MBSFN area is depicted in Fig. 1. Table I shows the values of the main parameters used in the system model simulations.

The video streaming simulator implemented is shown in Fig. 2. The link level is in charge of measuring the radio channel conditions of the users. Then, the system level calculates the BLER of each user every Transmission Time Interval (TTI).

Furthermore, Fig. 2 illustrates the application level configuration, where DASH [7] video segments are transmitted embedded as FLUTE protocol objects. The video is encoded and splitted into segments. It is considered that a video segment is correctly decoded if the failure probability in the decoding process is equal or less than $1 \%$.

The error probability of coded symbols can be calculated from the link level BLER, that is obtained from the Signal to Interference plus Noise Ratio (SINR) measurements for a given MCS. The probability of failure for the decoder $P\left(f_{R C}\right)$ has been calculated for each user and video segment in all the scenarios under study and is given as

$$
P\left(f_{R C}\right)=\sum_{n=0}^{k+r} P\left(f_{R C} \mid n\right) \times P(N=n)
$$

where $P\left(f_{R C} \mid n\right)$ is the failure probability of the decoder in case of receiving $n$ encoding symbols and $P(N=n)$ is the probability of correctly receiving $n$ symbols.

In (1) $P\left(f_{R C} \mid n\right)$ is calculated as [4] 
TABLE I

SYSTEM PARAMETERS

\begin{tabular}{c||c}
\hline \multicolumn{1}{c||}{ Parameter } & Value \\
\hline \hline MBSFN area size & $1 / 7$ / 19 eNodeBs \\
Interference model & 1 tier \\
eNodeBs geographical overlay & Hexagonal \\
ISD & $500 \mathrm{~m} / 1,732 \mathrm{~m}$ \\
Transmission power & $45 \mathrm{dBm}$ \\
Subframes reserved for MBMS & 1 \\
Cyclic prefix & Extended $(16.7 \mu \mathrm{s})$ \\
Bandwidth & $10 \mathrm{MHz}$ \\
Downlink base frequency & $2,110 \mathrm{MHz}$ \\
Pathloss model & $3 \mathrm{GPP}$ Urban Macrocell \\
Multipath channel model & ITU Pedestrian B \\
eNodeBs transmission antennas & 1 \\
Total number of UEs & 400 \\
UEs distribution & Uniform distribution \\
Modulation and Coding Schemes & MCS 4 / $/ 6 / 7$ / $/ 10 / 15$ \\
Length of Raptor Codes segments & $1 \mathrm{~s} / 2 \mathrm{~s} / 10 \mathrm{~s} / 20 \mathrm{~s}$ \\
\hline
\end{tabular}

$$
P\left(f_{R C} \mid n\right)= \begin{cases}1 & \text { if } n<k \\ 0.85 \times 0.567^{n-k} & \text { if } n \geq k\end{cases}
$$

On the other hand, the probability of success receiving $n$ symbols is modeled using a binomial distribution, given as

$$
P(N=n)=\left(\begin{array}{c}
k+r \\
n
\end{array}\right) \times(1-P E R)^{n} \times(P E R)^{(k+r-n)}
$$

where the Packet Error Rate (PER) is the probability of a symbol failure.

Since $P\left(f_{R C} \mid n\right)$ is the probability of failure when decoding an AL-FEC encoded DASH segment, it can be also considered as the probability of retransmitting this segment using a unicast HTTP transmission. By calculating $P\left(f_{R C} \mid n\right)$ for each segment and each user with different values of $k$ source symbols and $r$ repair symbols, it is possible to obtain the values of coverage for different AL-FEC code rates.

\section{PERformance EVAluation}

The performance evaluation has been carried out during a simulation time of 300 seconds (30,000 LTE frames) with 400 users uniformly distributed in the area, using 3 different deployments: 1-cell, 7 cell and 19-cell MBSFN area.

The utilization of different scenarios, where the size of the MBSFN area varies, is the basis for an analysis with different BLER distributions, in which the error probability decreases as the size of the MBSFN area increases. In addition, an evaluation of two different ISD deployments, ISD $=500 \mathrm{~m}$ and ISD $=$ $1,732 \mathrm{~m}$, is performed to study the improvements in coverage and performance obtained with small cells. Furthermore, a performance analysis using different AL-FEC block sizes is carried out to evaluate the benefits of longer segments. Finally, one of the results of this model is the possibility of evaluating

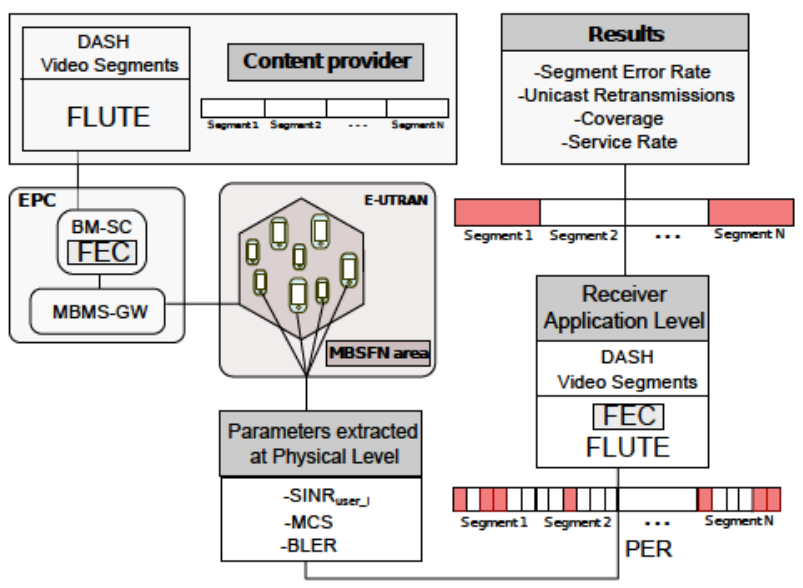

Fig. 2. Video streaming simulator architecture

the best combination of PHY-FEC and AL-FEC techniques to achieve the maximum service rate minimizing the number of retransmissions, enabling to evaluate the enhancements in a specific case for a video delivery service.

\section{A. Analysis of Coverage vs AL-FEC code rate}

An analysis of the coverage of users based on the AL-FEC code rate has been carried out for a deployment of 1-cell, 7cell and 19-cell MBSFN area. The multicast transmission has been performed using different MCSs.

Fig. 3a illustrates, for a deployment of a 7-cell MBSFN area with an ISD of $500 \mathrm{~m}$, the percentage of users that can correctly decode the video streaming service, based on the AL-FEC code rate using different MCSs. The improvement of the coverage of users can be observed both increasing the robustness of the MCS and the number of repair symbols of Raptor code, i.e. decreasing the code rate.

Fig. 3b shows the coverage of users based on the AL-FEC code rate using a fixed MCS of 15, in the 1-cell, 7-cell and 19-cell MBSFN area deployments. The coverage is enhanced introducing a single frequency network of 7 cells synchronized and cooperating together. Nevertheless, increasing the MBSFN area from 7-cell to 19-cell results in a slight improvement in coverage performance, at the cost of needing to coordinate a higher number of eNodeBs for multicast transmission.

\section{B. Analysis of Service Data Rate vs Coverage}

The use of AL-FEC increases the robustness and reliability of the service. However, the repair symbols that have been introduced affect the maximum service data rate versus coverage. Therefore, built on the coverage of users analysis for different AL-FEC code rate, the service data rate that can be achieved based on the coverage of users has been analyzed for 1-cell, 7-cell and 19-cell MBSFN area.

Fig. 4a shows, for a deployment of a 7-cell MBSFN area with an ISD of $500 \mathrm{~m}$, the service data rate versus the coverage for different MCSs. It can be observed that the utilization of less robust MCS, i.e. MCS 15, results in a higher service data rate under a certain level of coverage. However, in order to 


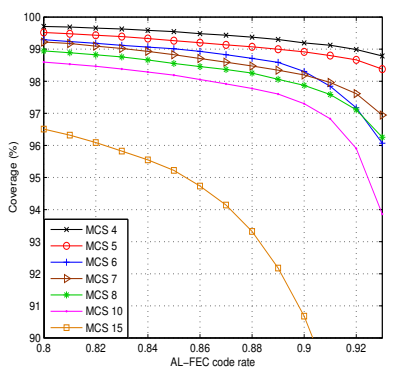

(a) 7-cell MBSFN area

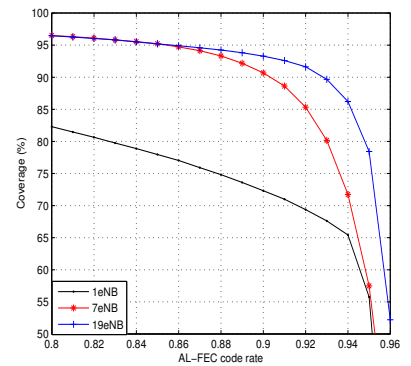

(b) MCS 15

Fig. 3. Coverage vs. AL-FEC code rate

minimize the number of unicast retransmissions, the coverage can be increased using more robust MCS and consequently reducing the service data rate.

This multicast transmission is using only one out of the six possible subframes that can be reserved for MBMS. Consequently, the service data rate results would be proportionally higher when using more subframes for the MBMS transmission.

Fig. $4 \mathrm{~b}$ shows the service data rate versus the coverage of users with a fixed MCS of 15 in the 1-cell, 7-cell and 19-cell MBSFN area deployments. It can be observed that the utilization of 7-cell MBSFN area noticeably increases the coverage of the users of the service. In addition, increasing the number of cells of the MBSFN area to 19, the service data rate and coverage are slightly improved.

One of the factors that has a direct impact on the radio channel conditions of the users is the ISD used for the cell deployment. The analysis of the performance using $500 \mathrm{~m}$ and 1,732 m ISD deployments illustrates the influence of cell size in the multicast service coverage.

Fig. 4c depicts, for a deployment of a 7-cell MBSFN area, the service data rate achieved based on the coverage of users with different MCSs and different ISD deployments. Note that the coverage achieved in the 1,732 $\mathrm{m}$ ISD deployment is poor in comparison with the coverage in the $500 \mathrm{~m}$ ISD deployment. This shows the importance of the size of the macro-cells in an urban environment, since it has a direct impact on the coverage of the service.

Live streaming services are intolerant to long delays. Due to this reason, a size of the video segments between 2 and 10 seconds is recommended. An evaluation of the performance using different size of the video segments is carried out.

Fig. 4d shows, for a deployment of a 7-cell MBSFN area with an ISD of $500 \mathrm{~m}$ and a fixed MCS of 15, that higher data rates can be achieved using longer protection periods. However, the benefit of using longer protection periods has the drawback of increasing the zap time and the transmission delay of the video streaming service. Note that increasing the size of the video segment to 20 seconds does not enhance the service data rate significantly.

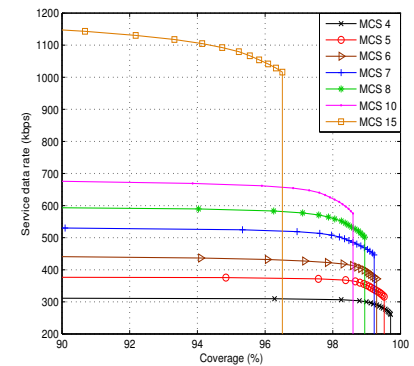

(a) 7-cell MBSFN area

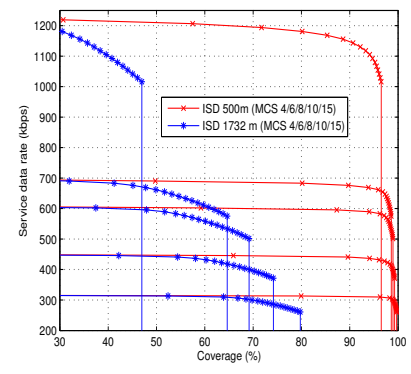

(c) Different ISD deployments

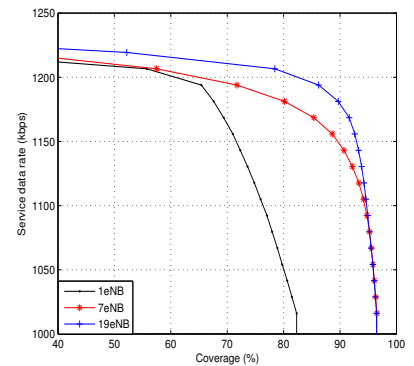

(b) MCS 15

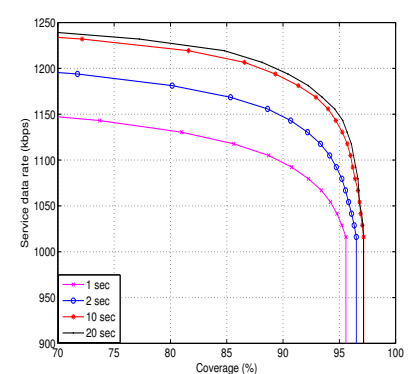

(d) Different AL-FEC segment length
Fig. 4. Service data rate vs. Coverage

\section{AL-FEC vs. PHY-FEC trade-off to maximize the Service Data Rate}

The final results of this study look for the AL-FEC and PHY-FEC combination to maximize the service data rate. In order to analyze this trade-off, an evaluation of the maximum service data rate based on a coverage of users higher than $90 \%$, has been carried out.

Fig. 5 shows, for a deployment with an ISD of $500 \mathrm{~m}$, the service data rate that can be achieved ensuring a coverage higher than $90 \%$ of the users, using the 1-cell, 7-cell and 19cell MBSFN area scenarios. Note that the service data rate that can be achieved in the 7-cell MBSFN area deployment is almost twice the service rate that can be obtained in the 1cell area deployment, when the coverage is between $90 \%$ and $96 \%$ of the users. On the other hand, the performance of $7-$ cell and 19-cell MBSFN area is almost the same. Thereby, the benefit of using a multi-cell MBSFN area instead of single-cell MBSFN is important in the service data rate and the coverage of users that can be achieved. However, the benefit of increasing the size of MBSFN area from 7-cell to 19-cell is not noticeable. MBSFN prevents interference and increases the strength of the received useful signal and, therefore, improves the perceived radio channel conditions of the users. This improvement implies that the SINR average measurement is increased and, therefore, the BLER at the receivers is decreased. Consequently, it is possible to use a less robust MCS in a multi-cell MBSFN deployment to improve the coverage of users demanding higher service data rate. Note that a small percentage of users cannot be served with the multicast transmission, due to their bad channel conditions, 


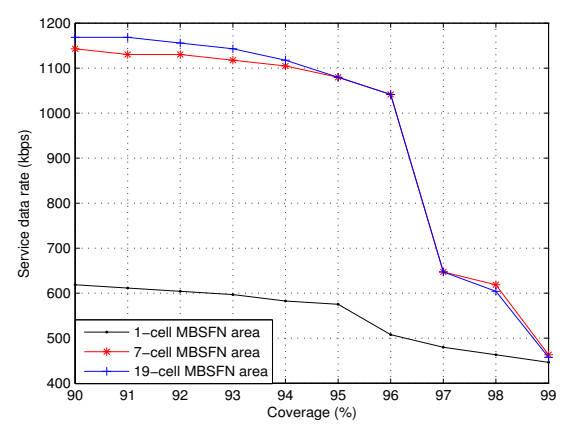

Fig. 5. Maximum data rate vs. Coverage for the AL-FEC/PHY-FEC trade-off

independently of the size of the MBSFN area deployed. The random distribution of users implies that some of them will generally be placed at the edge of the MBSFN area. Then, they will be served with a dedicated unicast retransmission.

\section{Specific case for a video streaming service}

The efficient utilization of resources using multicast transmissions has been studied for a specific case of a video streaming service. Table II shows the parameters used in the service deployment.

Fig. 6 depicts a comparison of the traffic generated using both multicast transmissions with unicast retransmissions of the lost blocks, with different MBSFN area size, and only unicast transmissions to every user demanding the service. It is worth noting that the traffic generated using only unicast transmissions is highly increased with the number of users. Alternatively, the use of multicast transmissions with unicast retransmissions improves significantly the efficiency in the utilization of resources in terms of the traffic generated, both per cell and per MBSFN area.

On the one hand, Fig. 6a shows that the traffic generated in a 7-cell MBSFN area is lower than in a 1-cell MBSFN area when the number of users in the area is greater than 30. Furthermore, in a 19-cell MBSFN area, the traffic is always higher than in a 7-cell MBSFN area, providing that the number of users demanding the service is the same in both scenarios.

On the other hand, Fig. 6b illustrates how the utilization of multi-cell MBSFN area yields an important reduction of the traffic generated per cell, whereas the use of 19-cell instead of 7-cell MBSFN area decreases slightly the traffic generated

TABLE II

PARAMETERS OF THE VIDEO STREAMING SERVICE

\begin{tabular}{c||c}
\hline Parameter & Value \\
\hline \hline Video bit rate & $1 \mathrm{Mbps}$ \\
ISD & $500 \mathrm{~m}$ \\
Modulation and Coding Scheme & 15 \\
AL-FEC code rate & 0.7874 \\
Length of AL-FEC segments & $2 \mathrm{~s}$ \\
Subframes reserved for MBMS & 1 \\
\hline
\end{tabular}

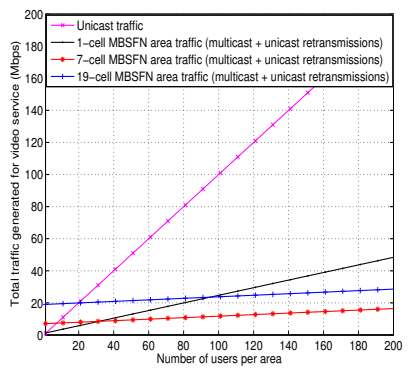

(a) Traffic per MBSFN area

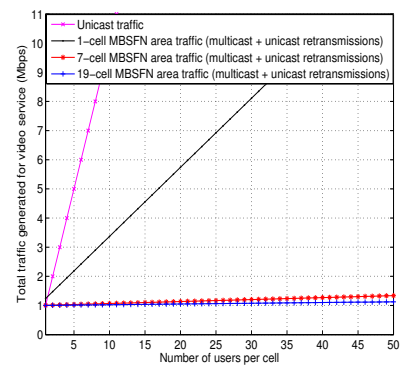

(b) Traffic per cell
Fig. 6. Comparison among traffic generated using MBSFN area with unicast retransmissions and unicast transmission for a video streaming service

per cell.

\section{CONCLUSiOnS}

This paper has presented an analysis of the impact of PHYFEC and AL-FEC techniques in the performance of a multicast video streaming service over a cellular network. This study is focused on the influence of multi-cell MBSFN area size to deploy a video streaming service. Several parameters, such as the MCS, the ISD between of the deployed eNodeBs and the AL-FEC code rate and protection period have been analyzed in order to provide the maximum service data rate and coverage of users. The results help to clarify the trade-off between the different parameters when the goal is to improve the quality of service by minimizing the number of unicast retransmissions. This kind of results will be useful for content providers and mobile network operators working in the deployment of a video streaming service.

\section{ACKNOWLEDGMENT}

This work was supported in part by the Spanish Ministry of Economy and Competitiveness, National Plan for Scientific Research, Development and Technological Innovation (INNPACTO subprogram), LTExtreme project (IPT-2012-0525430000).

\section{REFERENCES}

[1] "Introduction of the multimedia broadcast/multicast service (MBMS) in the radio access networks (RAN)-Stage 2”, 3GPP TS 25.346 v11.0.0, Sep 2012.

[2] "Evolved Universal Terrestrial Radio Access (E-UTRA) and Evolved Universal Terrestrial Radio Access Network (E-UTRAN); Overall description; Stage 2", 3GPP TS 36.300 v11.7.0, Sep 2013.

[3] "Multimedia Broadcast/Multicast Service (MBMS); Protocols and codecs”, 3GPP TS 26.346 v11.6.0, Sep 2013.

[4] C. Bouras, N. Kanakis, V. Kokkinos, and A. Papazois, "Application layer forward error correction for multicast streaming over lte networks,' International Journal of Communications Systems, 2012.

[5] J. Calabuig, J. Monserrat, D. Gozalvez, and D. Gomez-Barquero, "Alfec for streaming services in lte e-mbms," EURASIP Journal on Wireless Communications and Networking, 2013 2013:73.

[6] T. Stockhammer, A. Shokrollahi, M. Watson, M. Luby, and T. Gasiba, "Application layer forward error correction for mobile multimedia broadcasting," Handbook of Mobile Broadcasting: DVB-H, DMB, ISDB-T and Media Flo, CRC Press, pp. 239-280, 2008.

[7] ISO/IEC 23009-1 "Information technology - Dynamic adaptive streaming over HTTP (DASH) - Part 1: Media presentation description and segments formats”, April 2012. 